\title{
What We Can Find Beyond the Classic Neuroimaging Findings of Congenital Zika Virus Syndrome?
}

\author{
Bruno Niemeyer $^{a}$ Rafael Hollandab Bernardo Muniz ${ }^{b} \quad$ Edson Marchioric \\ anstituto Estadual do Cérebro Paulo Niemeyer and 3D Diagnóstico por Imagem/Hospital Casa de Portugal, Rio \\ de Janeiro, Brazil; ' ${ }^{b}$ Radiology Department, 3D Diagnóstico por Imagem/Hospital Casa de Portugal, Rio de Janeiro, \\ Brazil; ' Radiology Department, Universidade Federal do Rio de Janeiro, Rio de Janeiro, Brazil
}

\section{Keywords}

Congenital infection - Congenital Zika syndrome - Magnetic resonance imaging · Neuroinfectiology · Neuroimaging ·

Zika virus

\section{Abstract}

Background: In 1947, Zika virus (ZIKV) was first discovered in Monkeys, in Zika Forest, in Uganda, Africa. Five years later, (1952) the first human Zika infection was detected in Nigeria, Africa. After this date, only sporadic cases happened, until the first 3 epidemics occurred, all outside Africa. The first epidemic was in Yap Island in 2007, the second in French Polynesia in 2013, and the third in 2015 in the northeast of Brazil, and then the spread to the Americas in 2015 and 2016. However, it was only after the epidemic in the northeast of Brazil, in the first half of 2015, that many babies were born with microcephaly in the second semester of that same year and in 2016. Until now, every year, some babies are still born with congenital ZIKV syndrome (CZVS). Summary: The objective of this article is to describe infrequent and rarely discussed imaging (computed tomography and magnetic resonance imaging) findings of CZVS, in addition to those classically described such as a simplified gyral pattern, ven-

karger@karger.com

(c) 2020 S. Karger AG, Basel

www.karger.com/ene

Karger" triculomegaly, corpus callosum dysgenesis, craniofacial disproportion, and redundant scalp, thus suggesting an increase in the spectrum of neurological findings related to the syndrome.

(c) 2020 S. Karger AG, Basel

\section{Introduction}

Flaviviruses, transmitted by mosquitoes and ticks, are among the most important emerging viruses. After dengue, West Nile virus, and chikungunya, of which worldwide outbreaks were recorded in 1990, 1999, and 2013, respectively [1], Zika virus (ZIKV) is responsible for the most recent pandemic of arthropod-borne viral disease. ZIKV is an arbovirus of the Flaviviridae family, discovered in Uganda in 1947 and composed of ribonucleic acid; the first case in humans was described in 1952 [1-8]. It typically occurs in tropical and subtropical zones, with main African and Asian lineages originating from a common ancestor [1-8]. Like other arboviral diseases, ZIKV presents many barriers to the accumulation of mutations as a consequence of double replication in mammalian and invertebrate hosts, leading to relatively slow mutation fixation [5]. 
Since 2007, the distribution of ZIKV has broadened beyond its previous confinement to a narrow equatorial zone in Africa and Asia, with expansion to Micronesia and an epidemic in 2013-2014 in French Polynesia and New Caledonia. The disease has also spread to South and Central America and the Caribbean Islands [1, 4], aided substantially by the displacement of people and the presence of vectors. The first autochthonous transmission in Brazil was recorded in May 2015 [4, 8], with probable spread through the Pacific, as phylogenetic studies have confirmed a high degree of similarity $(99.7 \%$ for nucleotides and $99.9 \%$ for amino acids) with the virus circulating in the Pacific Ocean [2].

Brazil is the Latin American country most affected by ZIKV, with approximately $1,500,000$ cases reported between 2015 and 2016 and an increase of about 20 times in cases of microcephaly relative to previous years $[9,10]$. Likely contributors to the quick spread of ZIKV and its vector include global warming, climate change in association with El Niño, poverty, and a lack of population awareness, reflected clearly by the epidemic's intensity in the poor regions of northern and northeastern Brazil $[9,10]$. The number of reported ZIKV cases in Brazil declined from 205,000 in 2016 to 13,000 in 2017, likely due mainly to population immunity [10]. However, the continuous spread of the 4 main dengue serotypes in Brazil and throughout the Americas in recent decades suggests that ZIKV will continue to circulate, necessitating medical doctors' vigilance for compatible clinical findings [10].

Various forms of central nervous system (CNS) involvement associated with ZIKV infection, such as meningoencephalitis, Guillain-Barré syndrome, and acute disseminated encephalomyelitis, have been observed during epidemics $[2,3,5,7,11]$. In addition, a myriad of CNS malformation cases potentially related to congenital ZIKV infection, characterizing congenital ZIKV syndrome (CZVS), has attracted the attention of the scientific community and has been widely documented through computed tomography (CT) and magnetic resonance imaging (MRI). This article reviews these uncommon CT and MRI neuroimaging findings of CZVS.

\section{Congenital ZIKV Manifestations}

Intrauterine ZIKV infection signs are more severe when they appear in the first and second trimesters of pregnancy, primarily in the first trimester, and range from fetal death to numerous congenital abnormalities, including redundant skin on the scalp and neck (cutis gy- rata), low birth weight, polyhydramnios, anasarca, arthrogryposis (contracture of 2 or more joints since birth), hearing loss, and ocular and CNS malformations with craniofacial disproportion [3, 12-16]. Among congenital manifestations, microcephaly associated with ZIKV infection has had the greatest impact. It is well documented in Brazil and French Polynesia, in association with the Asian lineage of the virus. During the epidemic in Brazil occurring between March 2015 and February 2016, the number of microcephaly cases increased up to 20 times relative to previous years [3].

No consensus on the definition of microcephaly has been established in the literature. However, the condition is a brain growth disorder in which the cephalic perimeter is smaller than normal for the same age, sex, and race; cutoffs of below the $3 \mathrm{rd}$ and 5 th percentiles and $\leq 2$ and 3 SDs below the mean have been used [14-16]. Microcephaly can be caused by numerous factors, including genetic and infectious (rubella, cytomegalovirus, toxoplasmosis, herpes virus, and HIV) factors, as well as by exposure to drugs, alcohol, and other toxic substances in the environment. Clinically, patients show substantial neurological impairment, often with hypertonia, spasticity, and convulsions [16-21].

The pathogenesis of ZIKV-related microcephaly is not fully understood, but it is believed to begin with placental infection. Noronha et al. [22] reported the detection of viral proteins in Hofbauer cells and histiocytes in intervillous spaces, suggesting that ZIKV damages the placental barrier, inducing chronic placentitis, as documented in rats [23]. Subsequently, the infection disseminates to the fetal brain, where it infects progenitor neuronal cells, decreasing their viability and growth, resulting in the inhibition of cell proliferation and differentiation and in neuronal apoptosis, with consequent thinning of the cortex and the appearance of macroscopic aspects of microcephaly $[3,13]$. The placental inflammatory process has been suggested to act in synergy with cerebral ZIKV infection in the genesis of cerebral malformations [15]. Despite the neurotrophism of ZIKV, microcephaly has not been reported to date in children infected soon after birth, which can probably be explained by the low infectious potential of ZIKV when attacking mature neural cells $[3,15,16]$.

\section{Unusual Findings of CZVS}

\section{Infratentorial Parenchymal Calcifications}

Calcifications are common in CZVS, occurring in 88$100 \%$ of patients. Unlike the classic TORCH infections
Niemeyer/Hollanda/Muniz/Marchiori 


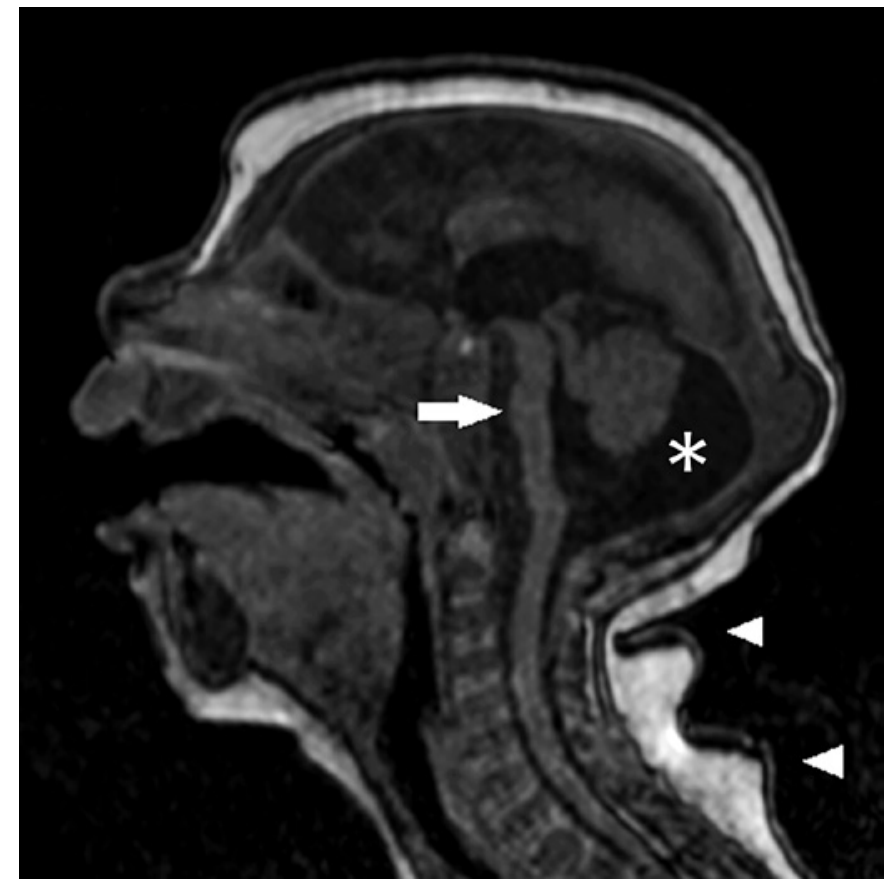

Fig. 1. Sagittal T1-weighted MRI slice without contrast showing hypoplasia of the pons (arrow), with loss of the usual convexity, in a 2-month-old patient with CZVS. Also shown are an enlarged cisterna magna $(*)$, reduced cerebellar volume, and excessive skin in the nuchal region (arrowheads).

(toxoplasmosis, other agents [e.g., HIV, syphilis] rubella, cytomegalovirus, and herpes simplex), in which calcifications are periventricular and cortical, ZIKV involvement tends to appear with cortical-subcortical transition, leading to the suggestion that it has a vascular infection component, as other processes that affect mainly this region are associated with vascular alterations [21, 24-32]. The infratentorial region is the least common site of involvement, affected in only $4-18 \%$ of cases; calcifications are seen more frequently in the basal nuclei/thalamus (29$65 \%)$, periventricular region (14-65\%), and cortical region (14-24\%) [21, 24-29, 33-35]. According to OliveiraSzejnfeld et al. [21], infratentorial calcifications are present in more severe cases associated with brainstem dysmorphia, aqueduct stenosis, and secondary hydrocephalus. Aragão et al. [31] corroborated this observation, reporting that infratentorial calcifications were more likely in the presence of arthrogryposis. On evolutionary follow-up, children with CZVS show reductions in the number, size, and density of calcifications, except those involving the basal and cerebellar nuclei [36]. CT is more sensitive than MRI, including magnetic susceptibility sequences, for the detection of calcifications [21, 24-29].

CZVS: Beyond the Classic Neuroimaging Findings

\section{Pontine and Cerebellar Hypoplasia}

Brainstem abnormalities are described in patients with congenital ZIKV infection, at frequencies of $21-70 \%$. They are characterized by a sharp, atrophic, encephalic trunk with preferential bridge involvement (Fig. 1), commonly related to greater disease severity and possibly related to a reduction in the number of descending fibers and direct viral damage [21, 24-35]. Other posterior fossa abnormalities include cerebellar hypoplasia (Fig. 2), which is usually diffuse and symmetrical and occurs in $27-82 \%$ of cases, and mega cisterna magna, which is related to greater disease severity; no direct relationship between these 2 manifestations has been established [21, 24-35]. Aragão et al. [31] reported that the presence of arthrogryposis was related to more severe brain damage, with a greater chance of cerebellar and cerebellum hypoplasia.

\section{Cerebellar Dysplasia}

Focal cerebellar cortical dysplasia is presumably due to a molecular defect or injury (i.e., hemorrhagic, viral, or toxic) occurring during the proliferation or migration phase of cerebellar development, with resulting disruption of cerebellar morphogenesis. It is present in some cases of CZVS characterized by early intrauterine infection [37]. The clinical manifestation of cerebellar dysplasia is variable, ranging from an incidental finding to severe neurological impairment. The MRI findings are abnormal foliation, obscurity of the gray-white matter junction, lack of normal white-matter arborization, cortical thickening, hemispheric hypertrophy, and cystlike cortical inclusions (Fig. 3).

Cerebellar cortical dysplasia in CZVS may be associated with microcephaly, which is currently recognized as only the "tip of the iceberg," one form of CZVS presentation [37]. As this finding has been described only recently, no study has examined the frequency of cerebellar cortical dysplasia in patients with CZVS, but it is believed to be rare; the first cases appeared in 2015, and cerebellar dysplasia alterations was recognized only in 2018 [37].

\section{Periventricular Septations}

An increase in the dimensions of the lateral ventricles is frequent in CZVS, occurring in $94-100 \%$ of cases and moderate/accentuated and symmetrical in the majority of patients. It has been associated with septations in only $10-29 \%$ of cases; when present, septations are commonly found in the occipital horns (Fig. 4) and are difficult to differentiate from periventricular cysts [21, 24-35]. 


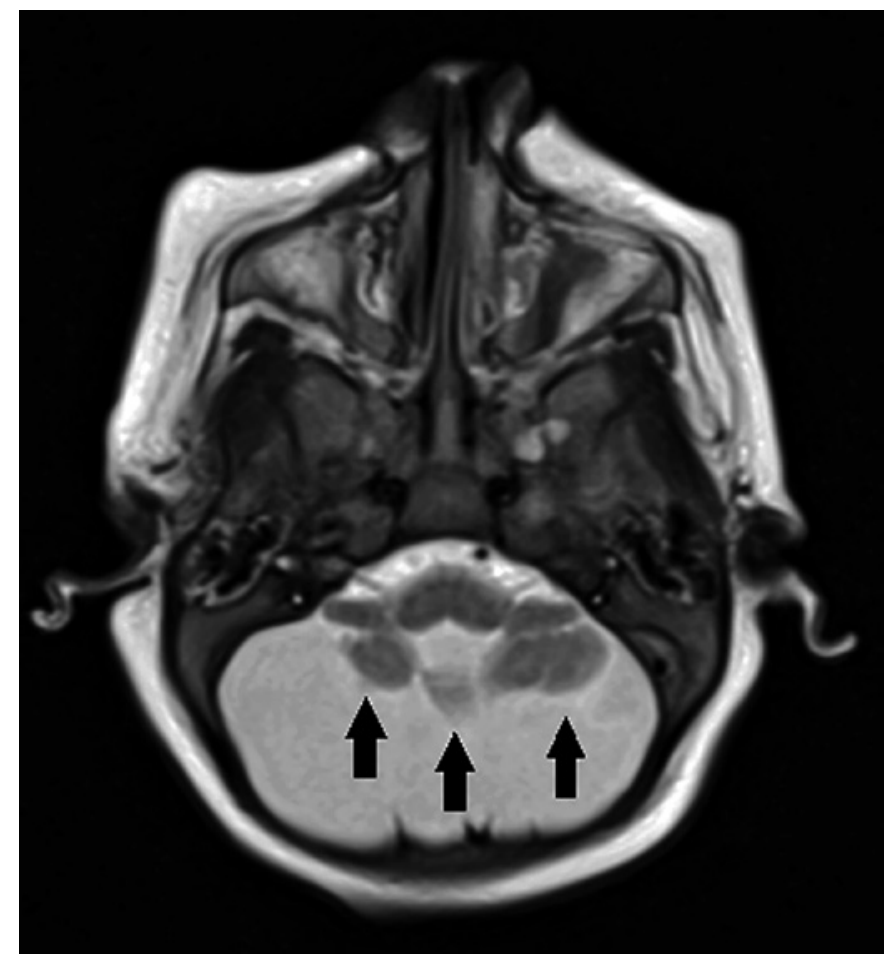

Fig. 2. Axial T2-weighted MRI slice showing diffuse, symmetrical cerebellar hypoplasia (arrows), with prominence of the CSF spaces in the posterior fossa, in a 2-month-old patient with CZVS.

\section{Hydrocephalus}

van der Linden et al. [38] described that hydrocephalus is a complication of SCZ in at least a proportion of patients. In this study, 14 of 24 children with CZVS (58\%) evolutionarily followed showed signs and symptoms suggestive of hypertensive hydrocephalus, characterized by vomiting, irritability, and/or sudden increases in head circumference percentile, with 2 of these patients (8\%) evolving with age-appropriate head circumference.

The evolution of the initial phenotype of microcephaly with ventriculomegaly to the later phenotype of severe hydrocephalus and probably high intracranial pressure is very likely multifactorial in nature. Considering that a relatively low-grade, persistent ZIKV infection in the CNS of surviving infants may exist, it would allow persistence of the pathologically induced apoptosis process over time. This candidate mechanism could be implicated in the observed progressive decrease in the size of the brain mass seen on follow-up CT scans, while the production of cerebrospinal fluid (CSF) remains active. Together, these mechanisms would lead to progressive enlargement of ventricular cavities, evolving toward the severe hydrocephalus. van der Linden et al. [38] also suggested that the progressive increase in CSF pressure may contribute additional harm to the CNS and cause stretching and loss of brain mass directly as a result of both its physical compressive effect and by opposing or overcoming blood perfusion pressures. In this context, the main mechanisms that physiologically regulate the turnover of CSF (including its production at choroid plexuses, venous absorption by dural Pachionian granulations, the inflow of CSF through the periarterial pathway toward the neuropil, and the outflow of CSF and interstitial fluid throughout the paravenous vessels pathways of the lymphatic system would hardly be impaired because of the progressive regression of brain mass structures as a whole. Additionally, the outflow that connects the paravenous system, with the dural lymphatic system draining into deep cervical lymph nodes, would likely also be impaired by the increased hydrostatic pressure determined by the CSF constraint [38].

\section{Polymicrogyria}

Abnormal cortical development is a frequent finding of CZVS, occurring in $94-100 \%$ of cases. It commonly presents as agyria and pachygyria, probably according to the phase of compromised cortical development [21,2435]. These findings are usually diffuse, with frontal lobe predomination, as well as various degrees of insular and parietal involvement, depending on disease severity. They are often associated with wide Sylvian and interhemispheric fissures and the enlargement of subarachnoid spaces, the latter associated in turn with brain volume loss and the impairment of CSF reabsorption [21, 24-35]. Cortical abnormalities in CZVS are better characterized by MRI than by CT [21, 24-29].

Polymicrogyria, defined as cortical surface irregularity due to an excessive number of abnormally small gyri (Fig. 5), is present in about $30 \%$ of CZVS cases, probably those characterized by late (i.e., 20th gestational week) intrauterine infection, and only in patients without microcephaly or with postnatal microcephaly $[30,39]$. This finding reinforces the characterization of polymicrogyria as a less-severe lesion, with consequent preservation of brain volume and the corpus callosum. In postnatal microcephaly, when polymicrogyria and pachygyria are found in the same infant, the former is located mainly in the frontal lobes and the latter is located in the posterior parts of the brain, mainly in the parietal and occipital lobes [39].

Currently, the spectrum of CZVS severity is divided into 3 manifestations: (1) microcephaly at birth, present- 

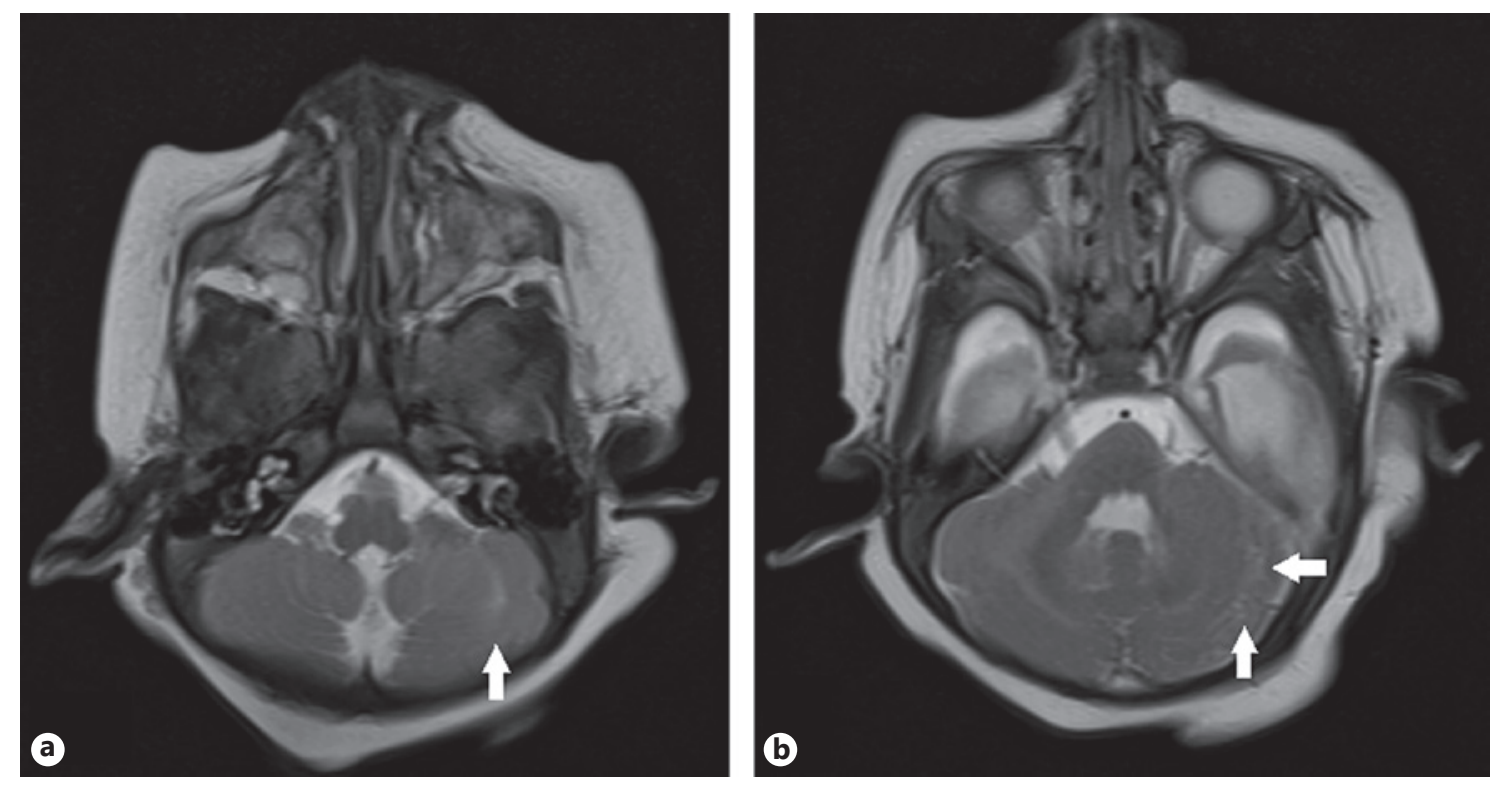

Fig. 3. a Axial T2-weighted MR image showing defective cerebellar foliation and irregularity of the gray-white matter junction in the left cerebellar hemisphere (white arrow) in a 4-month-old patient with CZVS. b Axial T2-weighted MR image showing cystlike inclusions in the left cerebellar hemisphere (white arrows) in the same patient. ing all abnormalities described in the literature and with a symmetrical appearance; (2) postnatal microcephaly, with no calcification outside of the cortical-subcortical junction and simplified giral pattern; and (3) restricted calcification at the cortical-subcortical junction, areas of polymicrogyria, delayed myelination, and slight asymmetrical ventricular enlargement, with no microcephaly $[30,39]$.

The presence of arthrogryposis is associated with the presence of pachygyria and/or simplified giral pattern and absence of polymicrogyria without statistical significance when compared with infants with CZVS without arthrogryposis [31]. This pattern suggests that CZVS with arthrogryposis occurs in the earlier stages of fetal development, as pachygyria is caused by the failure of neuronal migration and cortical organization around the 12-16th weeks of gestation, whereas polymicrogyria develops later, around the 20th week of gestation [31].

\section{Medullary and Nerve root Atrophy}

Although neuroimaging evaluation of CZVS focuses mainly on the cranium, spinal abnormalities, and nerve root involvement, as well as an apparent correlation between greater disease severity and the presence of arthrogryposis have been described recently [31]. Sagittal and axial T2-weighted MRI sequences show significant

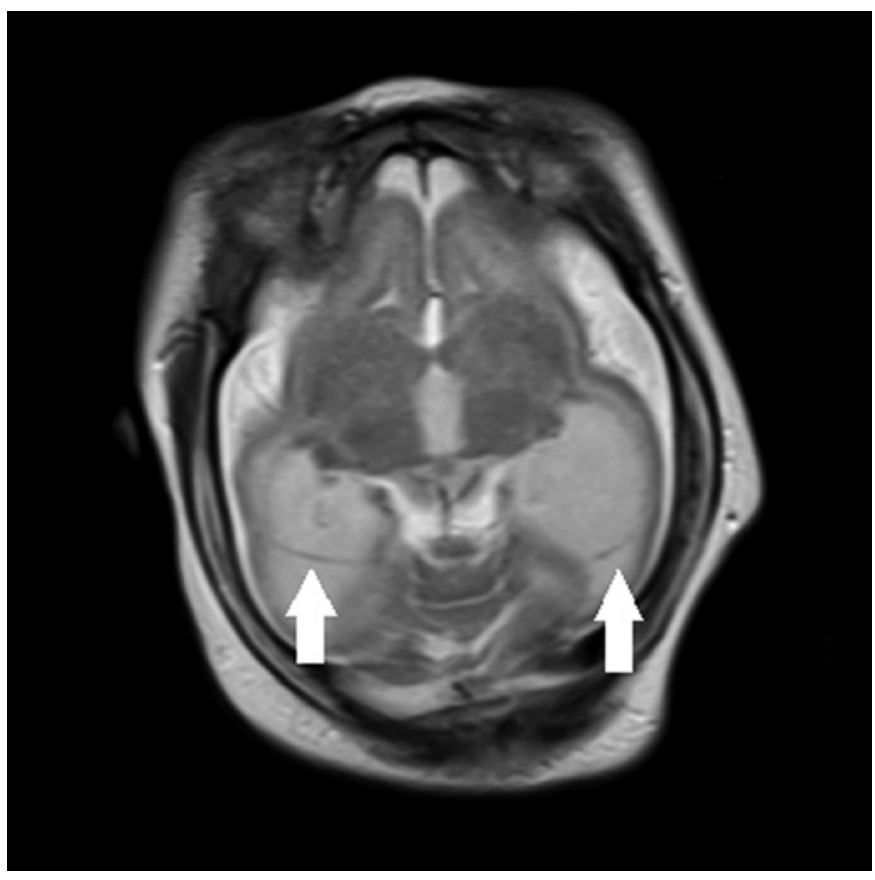

Fig. 4. Axial T2-weighted MR image showing thin septations in the posterior portions of the lateral ventricles (white arrows) in a 7-month-old patient with CZVS. 


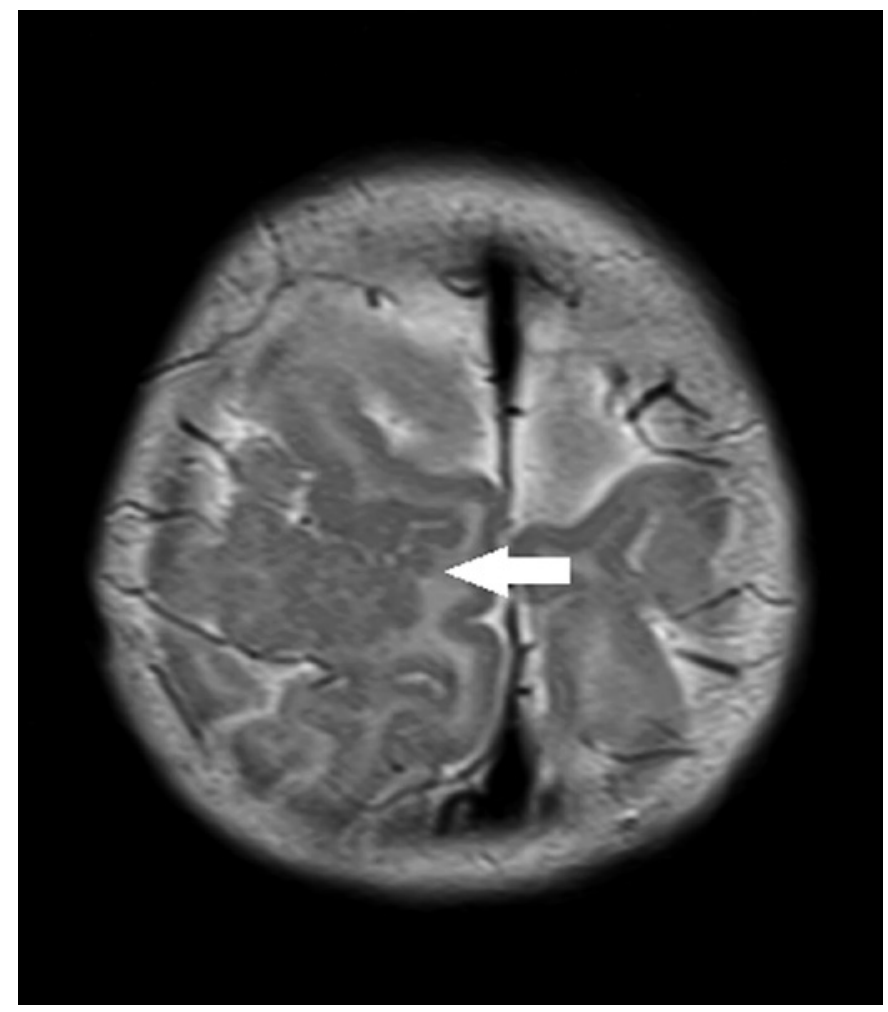

Fig. 5. Axial T2-weighted MR image showing an area of polymicrogyria in the right frontal lobe (white arrow) in a 7-month-old patient with CZVS.

diffuse reduced thickness in the spinal cord associated with substantial reduced thickness in the medullary cone anterior roots in patients with CZVS and arthrogryposis (Fig. 6). In patients without such joint alteration, MRI shows a reduction of spinal cord thickness only in the dorsal region and slight tapering of the anterior roots [31]. Mlakar et al. [12] also describe evident involvement of the descending anterior spinal cord tracts, with apparent preservation of the ascending posterior tracts.

\section{Cranial Nerve Enhancement}

Mulkey et al. [40] described a case of CZVS in a normocranial newborn, characterized by maternal infection around the 13th week of gestation, in which MRI performed 3 days after birth demonstrated the enhancement of cranial nerves III, V, VII, and VIII, with no other finding on non-contrast sequences. Cranial nerve enhancement on MRI is rarely seen in newborns and may be related to a postinfectious autoimmune disorder, with inflammation contributing to subsequent deficits, such as hearing loss. As gadolinium has not been used in the MRI evaluation of most reported cases of CZVS, the frequency of this finding cannot be estimated; such use of contrast may provide additional information on newborns exposed to Zika in the absence of non-contrast MRI findings. Future studies are likely to provide additional information, including the frequency of such findings in CZVS.

\section{Arterially Induced Ischemic Infarction}

Fetal or perinatal infarction may occur due to a variety of conditions, including placental abnormalities and neonatal and fetal conditions, with up to $40 \%$ of cases not clinically recognized at birth and subsequently discovered through imaging tests, especially in cases of neurodevelopmental delay. Although this finding in CZVS is questionable, Mulkey et al. [40] attributed their case of CZVS in a normocranial newborn to such a presentation, due to the absence of other maternal or birth risk factors that justified such a finding. Future studies are likely to provide additional information, including the frequency of such findings in CZVS.

\section{Conclusion}

Fetal ZIKV infection causes severe abnormalities in CNS development, and although the neuroimaging findings in CZVS are not pathognomonic, the diagnosis may be suggested in the presence of compatible clinical and laboratory data. The main findings of CZVS are craniofacial disproportion with a microcephalic aspect associated with calcifications, predominantly at the cortical-subcortical junction, cortex development malformations, ventriculomegaly, and abnormalities of the corpus callosum. Nevertheless, the spectrum of CZVS presentation includes findings other than those classically described; the search for such attributes should be part of the routine evaluation of imaging tests performed on children possibly affected by ZIKV.

Additionally, as recently described, we should be aware of evolutionary findings in CZVS, characterized by reduced cerebral calcifications and that some children are developing hydrocephalus.

\section{Acknowledgments}

We offer our deepest thanks to the institutions that provided technical support for the development and implementation of this study. 


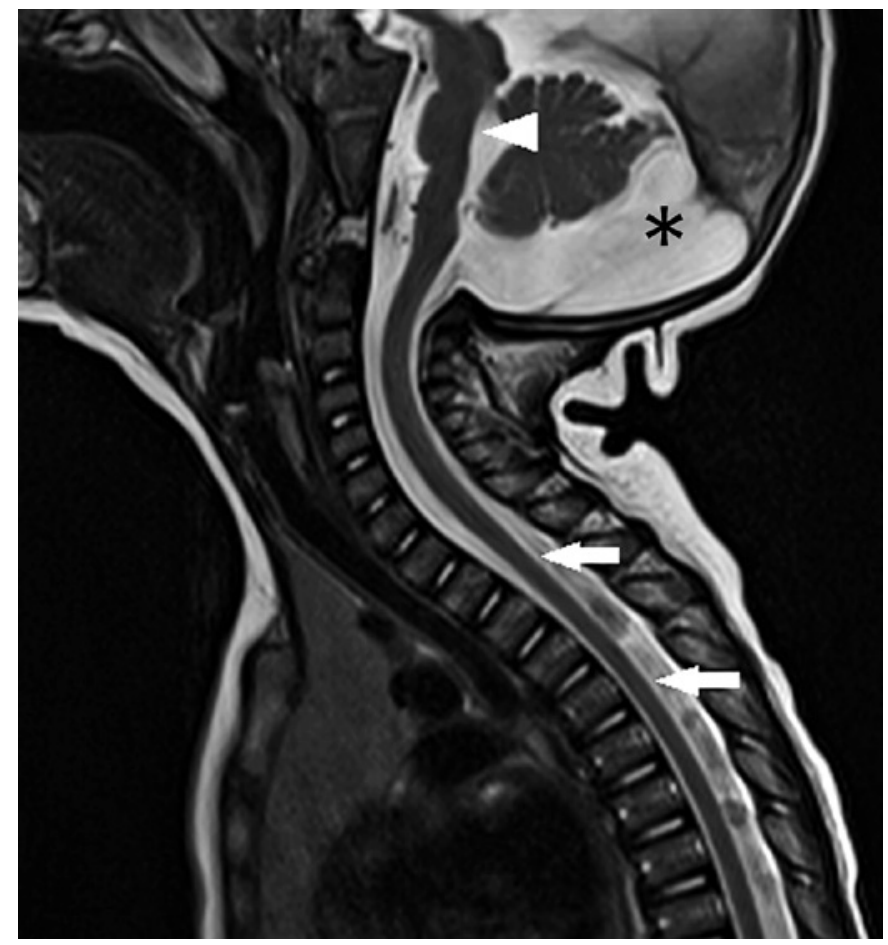

Fig. 6. A 14-month-old patient with CZVS. Sagittal T1-weighted MRI slice showing tapering of the dorsal medulla (arrows) in a 14-month-old patient with CZVS without arthrogryposis. Also shown are pontine hypoplasia (arrowhead) and an enlarged cisterna magna $(*)$.

\section{Statement of Ethics}

All procedures performed in studies involving human participants were in accordance with the ethical standards of the institutional and/or national research committee and with the $1964 \mathrm{Hel}-$ sinki declaration and its later amendments or comparable ethical standards.

\section{Disclosure Statement} close.

The authors report that there are no conflicts of interest to dis-

\section{Funding Sources}

This study was not funded.

\section{Author Contributions}

B.N. idealization and elaboration of the manuscript. R.H. elaboration of the manuscript. B.M. elaboration of the manuscript. E.M. manuscript revision.

\section{Informed Consent}

Informed consent was obtained from all individual participants included in the study.

\section{References}

1 Yadav S, Rawal G, Baxi M. Zika Virus: An Emergence of a New Arbovirus. J Clin Diagn Res. 2016 Jul;10(7):DM01-3.

2 Abushouk AI, Negida A, Ahmed H. An updated review of Zika virus. J Clin Virol. 2016 Nov; $84: 53-8$.

3 Atif M, Azeem M, Sarwar MR, Bashir A. Zika virus disease: a current review of the literature. Infection. 2016 Dec;44(6):695-705.

4 Aziz H, Zia A, Anwer A, Aziz M, Fatima S, Faheem M. Zika virus: global health challenge, threat and current situation. J Med Virol. 2017 Jun;89(6):943-51.

5 Blázquez AB, Saiz JC. Neurological manifestations of Zika virus infection. World J Virol. 2016 Nov;5(4):135-43.

6 Dick GW, Kitchen SF, Haddow AJ. Zika virus. I. Isolations and serological specificity. Trans R Soc Trop Med Hyg. 1952 Sep;46(5): 509-20.

7 Pinheiro TJ, Guimarães LF, Silva MT, Soares $\mathrm{CN}$. Neurological manifestations of Chikungunya and Zika infections. Arq Neuropsiquiatr. 2016 Nov;74(11):937-43.

8 Younger DS. Epidemiology of Zika Virus. Neurol Clin. 2016 Nov;34(4):1049-56.
9 Souza WV, Albuquerque MF, Vazquez E, Bezerra LC, Mendes AD, Lyra TM, et al. Microcephaly epidemic related to the Zika virus and living conditions in Recife, Northeast Brazil. BMC Public Health. 2018 Jan;18(1): 130-6.

10 Lowe R, Barcellos C, Brasil P, Cruz OG, Honório NA, Kuper H, et al. The Zika Virus Epidemic in Brazil: From Discovery to Future Implications. Int J Environ Res Public Health. 2018 Jan;15(1):96-113.

11 Niemeyer B, Niemeyer R, Borges R, Marchiori E. Acute Disseminated Encephalomyelitis Following Zika Virus Infection. Eur Neurol. 2017;77(1-2):45-6.

12 Mlakar J, Korva M, Tul N, Popović M, Poljšak-Prijatelj M, Mraz J, et al. Zika Virus Associated with Microcephaly. N Engl J Med. 2016 Mar;374(10):951-8.

13 Klase ZA, Khakhina S, Schneider AB, Callahan MV, Glasspool-Malone J, Malone R. Zika Fetal Neuropathogenesis: Etiology of a Viral Syndrome. PLoS Negl Trop Dis. 2016 Aug; 10(8):e0004877.

14 Wang JN, Ling F. Zika Virus Infection and Microcephaly: Evidence for a Causal Link. Int
J Environ Res Public Health. 2016 Oct;13(10): 1031-9.

15 Hajra A, Bandyopadhyay D, Heise LR, Bhadra R, Ball S, Hajra SK. Zika and pregnancy: A comprehensive review. Am J Reprod Immunol. 2017 Feb;77(2):1-7.

16 Ticconi C, Pietropolli A, Rezza G. Zika virus infection and pregnancy: what we do and do not know. Pathog Glob Health. 2016 Oct Dec;110(7-8):262-8.

17 Lammer EJ, Sever LE, Oakley GP Jr. Teratogen update: valproic acid. Teratology. 1987 Jun;35(3):465-73.

18 Shepard TH. "Proof" of human teratogenicity. Teratology. 1994 Aug;50(2):97-8.

19 Webster WS. Teratogen update: congenital rubella. Teratology. 1998 Jul;58(1):13-23.

20 Del Campo M, Jones KL. A review of the physical features of the fetal alcohol spectrum disorders. Eur J Med Genet. 2017 Jan;60(1):55-64.

21 Soares de Oliveira-Szejnfeld P, Levine D, Melo AS, Amorim MM, Batista AG, Chimelli L, et al. Congenital Brain Abnormalities and Zika Virus: What the Radiologist Can Expect to See Prenatally and Postnatally. Radiology. 2016 Oct;281(1):203-18. 
22 Noronha L, Zanluca C, Azevedo ML, Luz KG, Santos CN. Zika virus damages the human placental barrier and presents marked fetal neurotropism. Mem Inst Oswaldo Cruz. 2016 May;111(5):287-93.

23 Miner JJ, Cao B, Govero J, Smith AM, Fernandez E, Cabrera OH, et al. Zika virus infection during pregnancy in mice causes placental damage and fetal demise. Cell. 2016 May; 165(5):1081-91.

24 Aragao MF, van der Linden V, Brainer-Lima AM, Coeli RR, Rocha MA, Sobral da Silva P, et al. Clinical features and neuroimaging (CT and MRI) findings in presumed Zika virus related congenital infection and microcephaly: retrospective case series study. BMJ. 2016; 353:1901-10.

25 Hazin AN, Poretti A, Di Cavalcanti Souza Cruz D, Tenorio M, van der Linden A, Pena LJ, et al.; Microcephaly Epidemic Research Group. Computed Tomographic Findings in Microcephaly Associated with Zika Virus. N Engl J Med. 2016 Jun;374(22):2193-5.

26 Zare Mehrjardi M, Keshavarz E, Poretti A, Hazin AN. Neuroimaging findings of Zika virus infection: a review article. Jpn J Radiol. 2016 Dec;34(12):765-70.

27 Zare Mehrjardi M, Poretti A, Huisman TA, Werner $\mathrm{H}$, Keshavarz E, Araujo Júnior E. Neuroimaging findings of congenital Zika virus infection: a pictorial essay. Jpn J Radiol. 2017 Mar;35(3):89-94.

28 Moore CA, Staples JE, Dobyns WB, Pessoa A, Ventura CV, Fonseca EB, et al. Characterizing the Pattern of Anomalies in Congenital Zika Syndrome for Pediatric Clinicians. JAMA Pediatr. 2017 Mar;171(3):288-95.
29 Werner H, Sodré D, Hygino C, Guedes B, Fazecas T, Nogueira R, et al. First-trimester intrauterine Zika virus infection and brain pathology: prenatal and postnatal neuroimaging findings. Prenat Diagn. 2016 Aug;36(8): 785-9.

30 Aragão MF, Holanda AC, Brainer-Lima AM, Petribu NC, Castillo M, van der Linden V, et al. Nonmicrocephalic Infants with Congenital Zika Syndrome Suspected Only after Neuroimaging Evaluation Compared with Those with Microcephaly at Birth and Postnatally: How Large Is the Zika Virus "Iceberg"? AJNR Am J Neuroradiol. 2017 Jul;38(7): 1427-34.

31 Aragão MF, Brainer-Lima AM, Holanda AC, van der Linden V, Vasco Aragão L, Silva Júnior ML, et al. Spectrum of Spinal Cord, Spinal Root, and Brain MRI Abnormalities in Congenital Zika Syndrome with and without Arthrogryposis. AJNR Am J Neuroradiol. 2017 May;38(5):1045-53.

32 van der Linden V, Pessoa A, Dobyns W, Barkovich AJ, Júnior HV, Filho EL, et al. Description of 13 Infants Born During October 2015-January 2016 With Congenital Zika Virus Infection Without Microcephaly at Birth Brazil. MMWR Morb Mortal Wkly Rep. 2016 Dec;65(47):1343-8.

33 Ribeiro BN, Muniz BC, Gasparetto EL, Ventura N, Marchiori E. Congenital Zika syndrome and neuroimaging findings: what do we know so far? Radiol Bras. 2017 Sep-Oct; 50(5):314-22.
34 Ribeiro BN. Congenital Zika syndrome and neuroimaging findings. Radiol Bras. 2018 Mar-Apr;51(2):VII-VIII.

35 Peixoto Filho AA, de Freitas SB, Ciosaki MM, Oliveira LN, Dos Santos Júnior OT. Computed tomography and magnetic resonance imaging findings in infants with microcephaly potentially related to congenital Zika virus infection. Radiol Bras. 2018 Mar-Apr;51(2):119-22.

36 Petribu NC, Aragão MF, van der Linden V, Parizel P, Jungmann P, Araújo L, et al. Followup brain imaging of 37 children with congenital Zika syndrome: case series study. BMJ. 2017 Oct;359:j4188.

37 Ribeiro BN, Marchiori E. Congenital Zika syndrome associated with findings of cerebellar cortical dysplasia - Broadening the spectrum of presentation of the syndrome. J Neuroradiol. 2018 Nov;pii:S0150-9861(18)30261$\mathrm{X}$

38 van der Linden V, de Lima Petribu NC, Pessoa A, Faquini I, Paciorkowski AR, van der Linden $\mathrm{H} \mathrm{Jr}$, et al. Association of severe hydrocephalus with congenital Zika syndrome. JAMA Neurol. 2019 Feb;76(2):203-10.

39 de Freitas Ribeiro BN, Muniz BC, Gasparetto EL, Marchiori E. Congenital involvement of the central nervous system by the Zika virus in a child without microcephaly spectrum of congenital syndrome by the Zika virus. J Neuroradiol. 2018 Mar;45(2): 152-3.

40 Mulkey SB, Vezina G, Bulas DI, Khademian Z, Blask A, Kousa Y, et al. Neuroimaging Findings in Normocephalic Newborns With Intrauterine Zika Virus Exposure. Pediatr Neurol. 2018 Jan;78:75-8. 